\title{
Environment and Distribution of Age 0 Fishes in River Canard, a Lowland Ontario River
}

\author{
John K. LesLie and Charles A. Timmins \\ Department of Fisheries and Oceans, Great Lakes Laboratory for Fisheries and Aquatic Sciences, 867 Lakeshore Road, \\ Burlington L7R 4A6 Canada
}

Leslie, John K., and Charles A. Timmins. 2005 Environment and distribution of age 0 fishes in River Canard, a lowland Ontario river. Canadian Field-Naturalist 119(1): 16-25.

Age 0 fishes were collected to determine occurrence, relative abundance and species composition at three sites in River Canard, Ontario in spring-autumn, 1994-1995. This small lowland river (mean annual discharge, $3.2 \mathrm{~m}^{3} \mathrm{~s}^{-1}$ ) has variable flow during fish spawning and early nursery periods, high suspended particulate load, and sparse rooted vegetation and other physical cover. Forty-two taxa (12 families; 24544 specimens) collected with beach seines and a plankton net represented a wide range of reproductive strategies and a diverse taxocene. Gizzard Shad Dorosoma cepedianum (67\% of total catch), Orangespotted Sunfish Lepomis humilis (8\%), and Brook Silverside Labidesthes sicculus (6\%) were the most abundant species. Environmental conditions were such that fish attained autumnal lengths comparable to species in various systems throughout the ecoregion.

Key Words: age 0 fishes, abundance, growth, turbidity, lowland river, River Canard, Ontario.

The largest number of freshwater fish species in Canada inhabit tributary streams, marshes and near shore waters of Lake St. Clair and western Lake Erie. Despite more than 200 years of human interference throughout the area, the fish community maintains a surprisingly wide spectrum of feeding and reproductive guilds. Many species considered of minor importance to local and regional economies utilise an extensive network of low gradient, silt-laden streams and drainage systems that lace the landscape (Leslie and Timmins 1990; 1998a, 1998b; Leslie et al. 1999). The eventual movement of fishes from these waters to larger systems such as the Detroit River, Lake St. Clair, and Lake Erie contributes important fauna and nutrients to the lower Great Lakes basin (Herdendorf 1987).

Because many aspects of fish reproduction and early life history have large knowledge gaps, success of fish habitat conservation, enhancement and development rests largely on synopses of general ecology and anecdotal information. Nevertheless, in Canada, the Fisheries Act (Canada Department of Fisheries and Oceans [DFO] 1984) requires protection and enhancement of spawning, nursery, and residence habitat of all species, regardless of their economic importance and despite limited commitment to their study. Moreover, a thorough knowledge of the ecosystem is necessary for proper fisheries management and to improve our ability to determine the capacity of the system for sustainable development (Haffner 1992).

This paper describes the relative contribution and seasonality of age 0 fishes at three locations in River Canard, a tributary to the Detroit River. This study intended to provide information on features of early life history of species in small tributaries, and is the first such investigation in a lowland river in the lower Great Lakes.

\section{Study Area}

River Canard $\left(42^{\circ} 10^{\prime} 30^{\prime \prime} \mathrm{N}\right.$; $\left.83^{\circ} 05^{\prime} 00^{\prime \prime} \mathrm{W}\right)$ is a base gradient $\left(<0.4 \mathrm{~m} \mathrm{~km}^{-1}\right)$, meandering stream draining approximately $159 \mathrm{~km}^{2}$ in the St. Clair flats, Ontario. It is about $25 \mathrm{~km}$ long, with a mean annual discharge of $3.2 \mathrm{~m}^{3} \mathrm{~s}^{-1}$ (Ontario Ministry of Environment and Energy [MOEE] 1994). The drainage area is predominantly heavy textured, with poorly drained soils of dark grey spleisolic, with Devonian limestone dominating the bedrock (Chapman and Putnam 1984). Water quality is strongly influenced by runoff from intensely cultivated cropland that has a legacy of pesticide, herbicide and fertiliser application. In addition, the ecosystem is subjected to detrimental effects of intense vehicular traffic on several major highways. Agricultural and municipal drainage ditches connect River Canard with Lake Erie through Big and Cedar creeks and Lake St. Clair through the Pike Creek watershed (Figure 1).

Winner and Hartt (1969) described aspects of the aquatic biota of the river, exclusive of fishes. The rotifer Brachinus sp., an indicator of eutrophic systems, dominated the zooplankton community. In the lower river, common submersed vegetation included pondweed Potamogeton sp., Coontail Ceratophyllum demersum, Eurasian milfoil Myriophyllum sp., stonewort Chara sp., and Eelgrass Vallisneria americana. Dominant emergent plants were cattail Typha latifolia, $T$. angustifolia, bur-reed Sparganium sp., bulrush Scirpus sp., and sedges Carex spp. (Winner and Hartt 1969). This community of plants prevails, with the addition 
of American Lotus Nelumbo lutea, an exotic emergent established near the outlet to the Detroit River.

The general character of the river does not change drastically along its course; i.e., there are no pools or riffles, or vegetated backwaters. However, three sampling sites were chosen based on slight differences in physical and biological characteristics. Two sites (middle and upper reach) on the main course of the river and one (lower reach) near the confluence of a tributary to River Canard (Figure 1) were sampled for fishes. The upper reach (U: 4207'36"N; 8258'26"W), located about $14 \mathrm{~km}$ from the effluent, flows through pasture and scrubland. Stream width, typically $3 \mathrm{~m}$, and depth, 0.2-0.5 m, vary according to seasonal rainfall and flood control measures. In drought conditions, the upper reach is dry. Gravel substrate predominates in the centre, with sand and clay near gently sloping banks. Emergent plants consist mainly of Broad-leaved Cattail Typha latifolia, Common Reed Phragmites australis, and Great Bulrush Scirpus validus. Sparse riparian vegetation consists of small shrubs; e.g., Hazelnut Corylus americana and Dogwood Cornus racemosa. Partially submersed terrestrial grasses at the stream margin provide minimal cover and associated food for fishes. Crayfish Orconectes propinquus are abundant at all times. At least 20 drainage ditches are connected to the river between its origin and the upper reach sampling site.

The middle site (M: $\left.42^{\circ} 10^{\prime} 72^{\prime \prime} \mathrm{N} ; 83^{\circ} 02^{\prime} 05^{\prime \prime} \mathrm{W}\right)$ is situated on a tributary that meanders through cropland and pasture $0.2 \mathrm{~km}$ east of its confluence with River Canard. Midstream depth and width were $1 \mathrm{~m}$ and $11 \mathrm{~m}$, respectively, and the substrate consists of fine gravel and sand in the centre, with steep clay banks. No rooted aquatic plants are established, whereas trees, such as hawthorn Crataegus sp., Red Maple Acer rubrum, and Cottonwood Populus deltoides, form a closed canopy from mid-spring to late autumn.

In the lower reach (L: 42 ${ }^{\circ} 10^{\prime} 29^{\prime \prime} \mathrm{N}$; 8305'91"W), sampling took place $0.2 \mathrm{~km}$ from the Detroit River. At this location, River Canard is $230 \mathrm{~m}$ wide and about $1.0 \mathrm{~m}$ deep. Fish collections were taken at the margin of American Lotus, where grey clay is overlain with alluvium. Submersed macrophytes are sparse and scattered among debris of human origin. Broad-leaved Cattail, Giant Bulrush, and Common Reed are abundant at the shore.

\section{Methods}

In 1994, sampling took place only in the lower reach. Routine fish collections were taken weekly or twice monthly from late June to November. In 1995, collections began in mid-April and continued weekly or twice monthly until October, then once in November. Beach seines at the shore and a conical plankton net towed in open water were used to collect age 0 fishes. Most collections were made with a bagged larval fish seine (length $4 \mathrm{~m}$, width $1 \mathrm{~m}, 0.3-\mathrm{mm}$ Nitex mesh), hauled 10-15 m parallel to shore at maximum

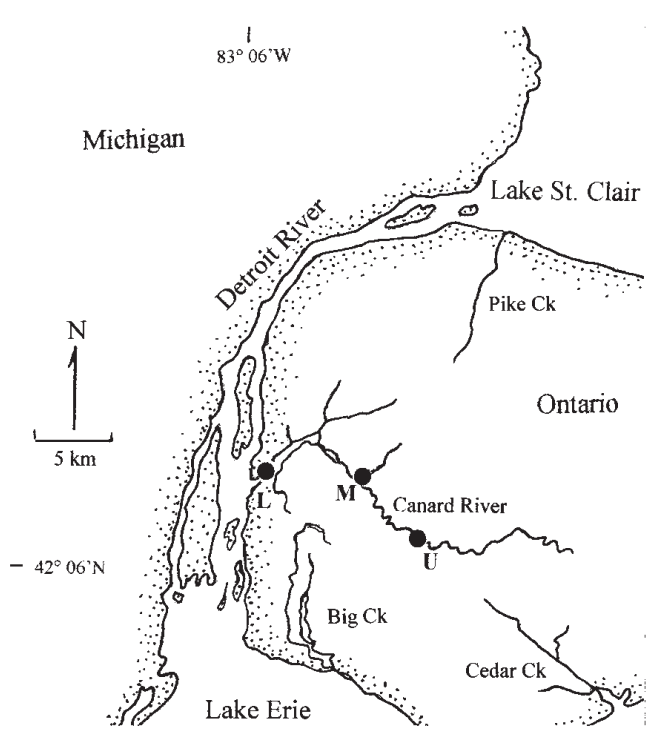

FIGURE 1. Map of study area, showing fish collection sites: $\mathrm{L}$ (lower reach), M (middle site), and U (upperreach) in River Canard, Ontario in 1994-1995.

wading depth of about $1.0 \mathrm{~m}$. The employment of this sampler is unique. At the end of each horizontal sweep, the seine is rapidly arced vertically to just above the surface, where contents are washed into its centre (Leslie et al. 1983). Each collection was immediately replicated at least once in the same location on each date. Specimens were immediately fixed with 5-20\% formalin. Additionally, a 6-m long, 1-m wide beach seine (6 $\mathrm{mm}$ mesh), used to provide ancillary information (species occurrence, seasonality) on age $1^{+}$ (juvenile and adult) fishes, swept a horizontal distance of about $15 \mathrm{~m}$ on 2-6 successive sampling efforts.

Twenty plankton tows were made in the lower reach to determine if species compositions in open water differed with those at the shore during usual peak occurrence of larvae in mid to late-June. Alternate tows were made upstream and downstream during the day over a constant distance of $230 \mathrm{~m}$ at speeds of $0.7-1.1 \mathrm{~m} / \mathrm{s}$ (average, $0.9 \mathrm{~m} / \mathrm{s}$ ). A $0.4-\mathrm{m}$ diameter, $1.5-\mathrm{m}$ long conical net $(0.4 \mathrm{~mm}$ Nitex mesh) collected fishes near the windward shore, the only habitat where depth was sufficient to perform linear hauls. The net was towed just below surface $10 \mathrm{~m}$ from the stern of an inflatable raft pushed by a small motor. Fish densities were expressed as number/100 $\mathrm{m}^{3}$. Sampling took place above a substrate of gravel, sand, and alluvium where maximum depth was $1.0-1.3 \mathrm{~m}$.

Jaccard's coefficient of community $C c$ (Oosting 1956) was used to compare longitudinal occurrences of fishes in the river. $C c=2 c / a+b$, where $a$ is the number of species at one site, $b$, the species in another, and $c$ is the number of species common to both sites. In addition, a turnover index $(T)$ measured assem- 
blage persistence in each site. $T=(C+E) /\left(S_{1}+S_{2}\right)$, where $C$ and $E$ are the number of species in respective collections at sampling site $S_{1}$ and $S_{2}$ (Przybylski 1994). Persistence $(P)$, an indicator of stochastic assemblages $=1-T$. All indices range from -1 to +1 ; a high value of $C c$ indicates a large number of species shared between sites. A high value of $P$ indicates high persistence and low fish species turnover. Common fishes were deemed species whose frequency of occurrence was $>50 \%$; uncommon occurred on $30-50 \%$ of sampling occasions, and rare fishes comprised the remainder.

All samples were processed in the laboratory within three months of collection. Specimens were placed in vials containing a $12: 1$ solution of $80 \%$ ethanol and glycerin, and stored in the dark at $22-25^{\circ} \mathrm{C}$. Measurements of total length (TL, $\mathrm{mm}$ ) were made on most-abundant fishes and those of special interest, such as rare taxa or recent invader. A Wild M5 dissecting microscope was used for length determinations, accurate to $0.1 \mathrm{~mm}$ for fish $<25 \mathrm{~mm}$ and $0.5 \mathrm{~mm}$ for larger specimens. For each sampling date, 10-66 specimens (average $=26$ ) of most-abundant species were measured. Field records included meteorological observations, water transparency (Secchi depth), water temperature, conductivity, and occasionally, $\mathrm{pH}$ and alkalinity. Environmental events, such as flooding or herbicide application on crops several days before sampling, may have affected the abundance of fishes. Thus, stream flow data on the sampling date and the previous two days were examined for possible concordance between flow volume and the total number of fishes found at each site.

\section{Results \\ Environmental characteristics}

In 1994 , water temperature averaged $22^{\circ} \mathrm{C}$ between June and early November, conductivity averaged 434 $\mu \mathrm{S} / \mathrm{cm}$ (range $=340-520 \mu \mathrm{S} / \mathrm{cm}$ ), and Secchi disc 0.15 to $0.50 \mathrm{~m}$ (mean $=0.26 \pm 0.13 \mathrm{~m})$. In 1995 , water temperature was maximum $\left(29.0^{\circ} \mathrm{C}\right)$ at $\mathrm{L}$ in early $\mathrm{Au}-$ gust, $25.0^{\circ} \mathrm{C}$ at $\mathrm{M}$ in mid-August, and $29.5^{\circ} \mathrm{C}$ at $\mathrm{U}$ in mid-June (Figure 2). Conductivity was highest and most variable at M, where readings of 1120 and 1800 $\mu \mathrm{S} / \mathrm{cm}$ were recorded in late June and September, respectively. These values coincided with some of the lowest flow volumes in the river $(0.012$ and 0.022 $\mathrm{m}^{3} / \mathrm{d}$ ). Conductivity was lower, and transparency higher at $\mathrm{L}$ than upstream, partly due to influx from the Detroit River. Eurasian milfoil first appeared in midMay, and cattail, bulrush, Common Reed, American Lotus and Purple Loosestrife, Lythrum salicaria, were abundant in late July. Prolonged drought was responsible for a series of isolated pools (depth, 0.1-0.3 m) that developed at U in late June-early July. Spearman's rank correlation $\left(\mathrm{r}_{\mathrm{s}}\right)$ analyses indicated total fish abundance and four environmental variables on sampling dates were not correlated (Table 1), nor were flow volume and abundance correlated on both days prior to sampling $\left(\mathrm{r}_{\mathrm{s}}=-0.02\right.$ to 0.20$)$.

\section{Overall relative abundance and occurrence}

All reproductive guilds in Canada (Balon 1975) were represented in the age 0 assemblage of 42 species, 12 families (Table 2), and total collection of 24544 fishes. Clupeids (67\%), dominated by Gizzard Shad, were the most abundant species in both 1994 and 1995; the remainder of the catch consisted mainly of centrarchids (13\%), an atherinid (Brook Silverside, 6\%) and 14 cyprinid species (6\%). Bowfin, Spotfin Shiner, Banded Killifish, Blackside Darter, Tadpole Madtom, and Round Goby were collected only as age $1^{+}$fishes. Several non-indigenous species (see Table 2 for scientific names), classified "Vulnerable" (Campbell 2001), were also found.

Twelve common species were recorded, although only three contributed $>5 \%$ to total catch (Table 2). Common Carp and Orangespotted Sunfish were the only common fishes among seven introduced species, including Alewife, Bigmouth Buffalo, Goldfish, White Perch and Round Goby. Maximum fish abundance occurred at all sites on the same date (23 June) and in similar environmental conditions in 1994 and 1995 (Figure 3): low, declining flow (0.01-0.05 m³/d), low transparency, high conductivity, medium-high water temperature $\left(23.5\right.$ to $\left.29.5^{\circ} \mathrm{C}\right)$, and in $\mathrm{L}$, surface emergence of American Lotus.

Gizzard Shad was the most abundant age 0 fish at $\mathrm{L}$ and $\mathrm{M}$, and centrarchids at $\mathrm{U}$, where Orangespotted Sunfish alone represented $68.3 \%$ of total catch at that site. Bluntnose Minnow, Orangespotted Sunfish, and Quillback were the only species more abundant in $\mathrm{U}$ than downstream. White Suckers were exclusive to $\mathrm{M}$, whereas Goldfish were caught only at U (Table 2).

Frequency of occurrence of all taxa averaged $35 \%$ in L, compared with $28 \%$ and $22 \%$ in $\mathrm{M}$ and $\mathrm{U}$, respectively. Most species, including several listed as "vulnerable" in Canada: Bigmouth Buffalo, Pugnose Shiner, Pugnose Minnow, Central Stoneroller, and Longear Sunfish, were caught sporadically and in low number. Between-site species turnover of age 0 fishes differed according to sampling dates, ranging from 0.9 in early August at $\mathrm{L}$ and $\mathrm{M}$, to 1.0 (complete lack of persistence) at $\mathrm{L}$ and $\mathrm{U}$ in early June.

Overall species richness increased by a factor of 2.2 from upper to lower reach. Assemblages of age 0 fishes were similar taxonomically at all sites only in the first few weeks in June ( $\mathrm{Cc}=0.33$ to 0.46$)$. Generally, lowest persistence, or highest turnover, also took place in mid-June. Highest coefficient of community (0.5) was recorded in mid-June, when many taxa intermingled at all sites. The middle site and $\mathrm{U}$ usually shared fewer species than $\mathrm{M}$ and L. Fish cooccurrence, both between $\mathrm{L}$ and $\mathrm{M}$ and $\mathrm{L}$ and $\mathrm{U}$, were minimum in early August $(\mathrm{Cc}=0.13$ and 0.08 , respectively), and $\mathrm{M}$ and $\mathrm{U}$ in early July (0.07). 

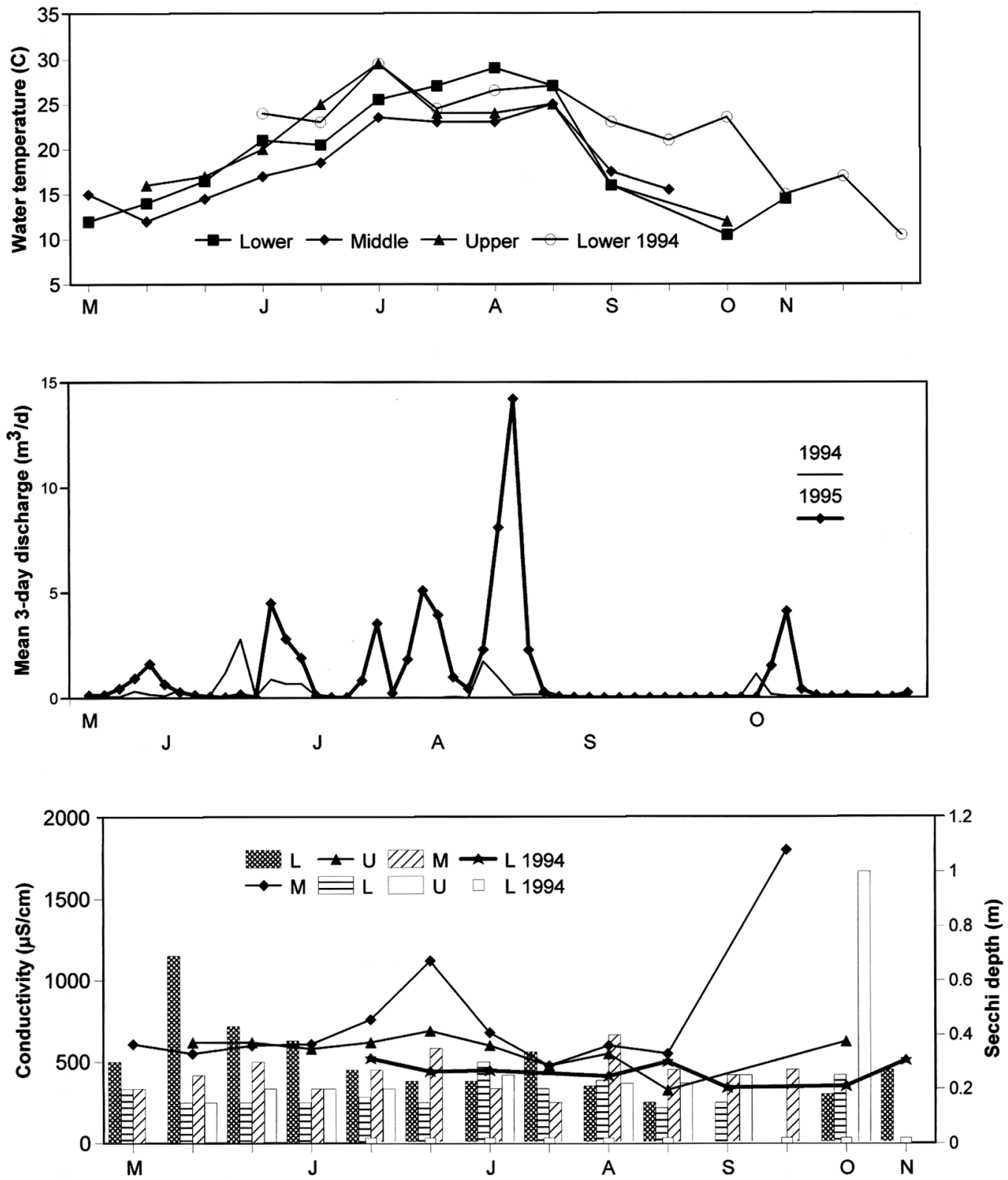

FIGURE 2. Environmental variables for River Canard: water temperature at three sites (upper), mean 3-day discharge (middle), and conductivity and Secchi depth (transparency) (lower graph).

\section{Fish Seasonality}

Upper reach

Abundance and frequency of occurrence of age 0 species (17 taxa) were consistently low (Figure 3). A single Central Mudminnow (18 mm TL) was the only age 0 fish found in late May. Quillback (15.9 $0.8 \mathrm{~mm}$; $\mathrm{N}=42$ ) next appeared (early June), but was not record- ed after late June. Johnny Darters (9-11 mm), which first appeared in early June, contributed $7.4 \%$ to the total catch (1839 fishes). Small numbers of recently hatched Common Carp (5-7 $\mathrm{mm})$ were also caught in early June, but were not found after mid-July. No adult Gizzard Shad were caught on any date. However, Shad larvae were found sporadically until early Sep- 
TABLE 1. Spearman's rank correlation coefficients for overall fish abundance relative to environmental variables in River Canard. Upper row denotes 1994 values. Number of measurements in parentheses.

\begin{tabular}{lccc}
\hline \hline Variable & Lower reach & Middle site & Upper reach \\
\hline Conductivity & $0.539(12)$ & & \\
& $0.154(9)$ & $0.212(10)$ & $0.381(8)$ \\
Secchi depth & $0.150(10)$ & & \\
& $0.142(9)$ & $-0.433(10)$ & $0.226(8)$ \\
Temperature & $0.523(12)$ & & \\
& $-0.233(9)$ & $0.422(10)$ & $-0.536(8)$ \\
Flow volume & $-0.119(12)$ & & \\
& $-0.300(9)$ & $-0.139(10)$ & $-0.429(9)$ \\
\hline \hline
\end{tabular}

tember, when the largest specimen $(94 \mathrm{~mm})$ was collected. As flow volume decreased to $0.01 \mathrm{~m}^{3} / \mathrm{d}$ in late June, an isolated pool $\left(\sim 350 \mathrm{~m}^{2}\right)$ developed at the sampling site. Eight species and $81 \%$ of the total collection were caught in this pool on 23 June. Orangespotted Sunfish was the sole recurring adult fish.
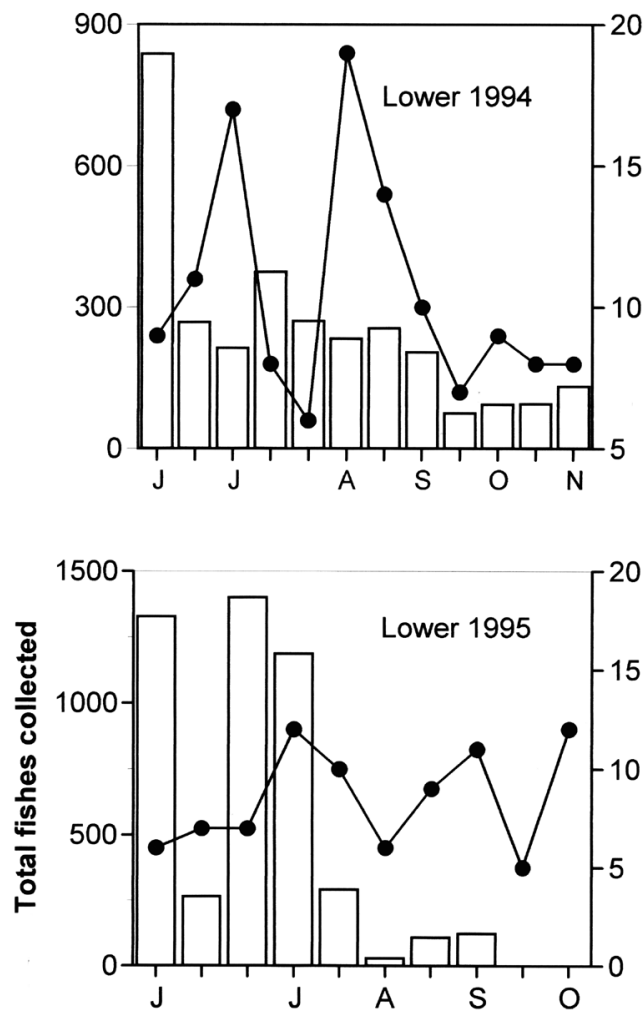

\section{Middle site}

Fishes represented 18 species and 7 reproductive guilds at the middle site. Non-guarding phytophils (Common Carp, Bigmouth Buffalo) and guarding nestspawners, such as psammophils Bluntnose Minnows, Black Crappie, numerically dominated the assemblage. Highest catch and number of species were observed in late June (Figure 3). Logperch were first caught in mid-May and Gizzard Shad, Yellow Perch, White Sucker, and Quillback in early June. Common Carp (9.4 \pm $2.6 \mathrm{~mm} ; \mathrm{N}=23$ ), Bluegill, and Emerald Shiner next appeared in late June. Gizzard Shad, which represented $78.5 \%$ of the total collection (4744 fishes), was the only highly persistent species (Table 3 ). Although various age 0 and $1+$ fishes co-occurred in early spring and autumn, they were usually temporally separate during the early summer nursery period. Emerald Shiner was the only adult fish caught repeatedly.

\section{Lower reach}

Although sampling in 1994 began in late June, 12 taxa were found that were not caught in 1995, when collections began in April. However, these fishes con-
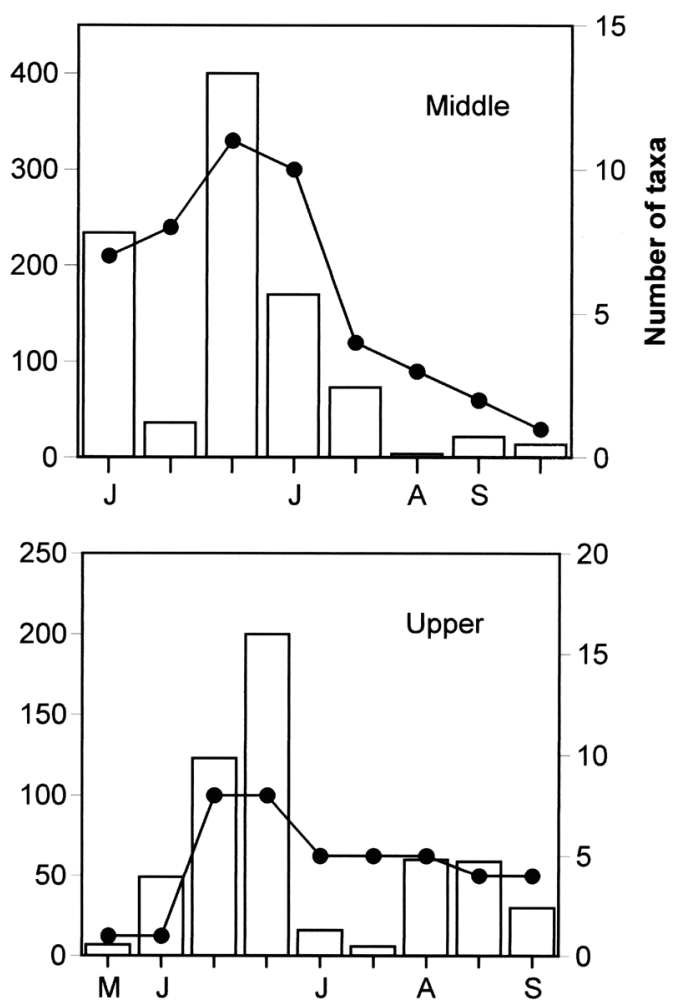

FIGURE 3. Seasonal total catch (bar) and number (line) of age 0 fishes found in River Canard, 1994-1995. 
TABle 2. Age 0 fish (0) species in River Canard lower (L), middle (M), and upper (U) sites, 1994-1995. 1+ indicates occurrence of juveniles $>1$ year old, or adults. $f_{o}=$ relative overall occurrence: $\mathrm{C}$ common, $\mathrm{U}$ uncommon, $\mathrm{R}$ rare. Guild = reproductive classification (Balon 1975): Ph phytophil, Lp lithopelagophil, Pl phytolithophil, Ps psammophil, Pe pelagophil, Sp speleophil, P polyphil. Total catch 24544. TL (mm) indicates smallest measured specimen of each species. Species status: N Native, I introduced, V "vulnerable". Species names follow Mandrak and Crossman (1992).

\begin{tabular}{|c|c|c|c|c|c|c|c|c|c|}
\hline Species & Common name & Status & $\mathrm{L}$ & M & $\mathrm{U}$ & Guild & $\mathrm{f}_{\mathrm{o}}$ & Catch $(\%)$ & $\mathrm{TL}$ \\
\hline Lepisosteus osseus & Longnose Gar & $\mathrm{N}$ & 0 & & 0 & $\mathrm{Ph}$ & $\mathrm{R}$ & & 80 \\
\hline Amia calva & Bowfin & $\mathrm{N}$ & $1+$ & & & $\mathrm{Ph}$ & $\mathrm{R}$ & & \\
\hline Dorosoma cepedianum & Gizzard Shad & $\mathrm{N}$ & $0,1+$ & 0 & 0 & $\mathrm{Lp}$ & $\mathrm{C}$ & 66.6 & 4 \\
\hline Alosa pseudoharengus & Alewife & $\mathrm{I}$ & 0 & & & $\mathrm{Pl}$ & $\mathrm{R}$ & & 34 \\
\hline Esox lucius & Northern Pike & $\mathrm{N}$ & $0,1+$ & $1+$ & $0,1+$ & $\mathrm{Ph}$ & $\mathrm{R}$ & & 102 \\
\hline Umbra limi & Central Mudminnow & $\mathrm{N}$ & & & 0 & $\mathrm{Ph}$ & $\mathrm{R}$ & & 18 \\
\hline Moxostoma macrolepidotum & Shorthead Redhorse & $\mathrm{N}$ & 0 & & & $\mathrm{Li}$ & $\mathrm{R}$ & & 74 \\
\hline Moxostoma sp. & Redhorse sp. & $\mathrm{N}$ & 0 & 0 & & $\mathrm{Li}$ & $\mathrm{R}$ & & \\
\hline Catostomus commersoni & White Sucker & $\mathrm{N}$ & & $0,1+$ & & $\mathrm{Li}$ & $\mathrm{R}$ & & 18 \\
\hline Ictiobus cyprinellus & Bigmouth Buffalo & $\mathrm{I}, \mathrm{V}$ & 0 & 0 & 0 & $\mathrm{Ph}$ & $\mathrm{U}$ & & 60 \\
\hline Carpiodes cyprinus & Quillback & $\mathrm{N}$ & $0,1+$ & 0 & $0,1+$ & Ps & $\mathrm{C}$ & 1.1 & 6 \\
\hline Notropis atherinoides & Emerald Shiner & $\mathrm{N}$ & $0,1+$ & $0,1+$ & $1+$ & $\mathrm{Pe}$ & $\mathrm{C}$ & 2.5 & 6 \\
\hline Notropis hudsonius & Spottail Shiner & $\mathrm{N}$ & $0,1+$ & $0,1+$ & $0,1+$ & Ps & $\mathrm{C}$ & 0.5 & 11 \\
\hline Notropis volucellus & Mimic Shiner & $\mathrm{N}$ & $0,1+$ & $0,1+$ & & $\mathrm{Pl}$ & $\mathrm{R}$ & & 23 \\
\hline Notropis stramineus & Sand Shiner & $\mathrm{N}$ & 0 & & & Ps & $\mathrm{R}$ & & 23 \\
\hline Luxilus cornutus & Common Shiner & $\mathrm{N}$ & $0,1+$ & & $1+$ & $\mathrm{Li}$ & $\mathrm{R}$ & & 35 \\
\hline Notropis anogenus & Pugnose Shiner & $\mathrm{N}, \mathrm{V}$ & $0,1+$ & & & $\mathrm{Li}$ & $\mathrm{R}$ & & 30 \\
\hline Opsopoeodus emiliae & Pugnose Minnow & $\mathrm{N}, \mathrm{V}$ & $0,1+$ & & & $\mathrm{Ph}$ & $\mathrm{R}$ & & 22 \\
\hline Notemigonus crysoleucas & Golden Shiner & $\mathrm{N}$ & 0 & & $1+$ & $\mathrm{Ph}$ & $\mathrm{R}$ & & 26 \\
\hline Cyprinus carpio & Common Carp & $\mathrm{I}$ & $0,1+$ & $0,1+$ & $0,1+$ & $\mathrm{Ph}$ & $\mathrm{C}$ & 1.0 & 5 \\
\hline Carassius auratus & Goldfish & I & $1+$ & & 0 & $\mathrm{Ph}$ & $\mathrm{R}$ & & 24 \\
\hline Cyprinella spiloptera & Spotfin Shiner & $\mathrm{N}$ & $1+$ & & & $\mathrm{Pl}$ & $\mathrm{C}$ & & 26 \\
\hline Campostoma anomalum & Central Stoneroller & $\mathrm{N}, \mathrm{V}$ & & & 0 & $\mathrm{Li}$ & $\mathrm{R}$ & & \\
\hline Pimephales notatus & Bluntnose Minnow & $\mathrm{N}$ & $0,1+$ & $0,1+$ & $0,1+$ & $\mathrm{Sp}$ & $\mathrm{C}$ & 1.3 & 8 \\
\hline Pimephales promelas & Fathead Minnow & $\mathrm{N}$ & $0,1+$ & $0,1+$ & $0,1+$ & Ps & $\mathrm{R}$ & & 21 \\
\hline Ameiurus nebulosus & Brown Bullhead & $\mathrm{N}$ & $0,1+$ & & $1+$ & $\mathrm{Sp}$ & $\mathrm{R}$ & & 42 \\
\hline Ictalurus punctatus & Channel Catfish & $\mathrm{N}$ & $0,1+$ & & & $\mathrm{Sp}$ & $\mathrm{R}$ & & 53 \\
\hline Noturus gyrinus & Tadpole Madtom & $\mathrm{N}$ & & & $1+$ & $\mathrm{Sp}$ & $\mathrm{R}$ & & \\
\hline Fundulus diaphanus & Banded Killifish & $\mathrm{N}$ & $1+$ & & & $\mathrm{Ph}$ & $\mathrm{R}$ & & 47 \\
\hline Morone chrysops & White Bass & $\mathrm{N}$ & 0 & & & $\mathrm{Pl}$ & $\mathrm{R}$ & & 37 \\
\hline Morone americana & White Perch & I & 0 & & & $\mathrm{Pl}$ & $\mathrm{R}$ & & 56 \\
\hline Lepomis gibbosus & Pumpkinseed & $\mathrm{N}$ & $0,1+$ & & & $\mathrm{P}$ & $\mathrm{R}$ & & \\
\hline Lepomis megalotis & Longear Sunfish & $\mathrm{N}$ & 0 & & & $\mathrm{Li}$ & $\mathrm{R}$ & & 28 \\
\hline Lepomis cyanellus & Green Sunfish & $\mathrm{N}$ & $0,1+$ & & $0,1+$ & $\mathrm{Li}$ & $\mathrm{R}$ & & 40 \\
\hline Lepomis humilis & Orangespotted Sunfish & I, V & $0,1+$ & $0,1+$ & $0,1+$ & $\mathrm{Li}$ & $\mathrm{C}$ & 8.4 & 5 \\
\hline Lepomis macrochirus & Bluegill & $\mathrm{N}$ & $0,1+$ & $0,1+$ & $0,1+$ & $\mathrm{Li}$ & $\mathrm{C}$ & 3.5 & 3 \\
\hline Ambloplites rupestris & Rock Bass & $\mathrm{N}$ & 0 & & & $\mathrm{Li}$ & $\mathrm{R}$ & & 38 \\
\hline Micropterus salmoides & Largemouth Bass & $\mathrm{N}$ & $0,1+$ & & & $\mathrm{Ph}$ & $\mathrm{R}$ & & 17 \\
\hline Micropterus dolomieu & Smallmouth Bass & $\mathrm{N}$ & $0,1+$ & & & $\mathrm{Li}$ & $\mathrm{R}$ & & \\
\hline Pomoxis nigromaculatus & Black Crappie & $\mathrm{N}$ & $0,1+$ & $0,1+$ & 0 & $\mathrm{Ph}$ & $\mathrm{C}$ & 1.1 & 7 \\
\hline Pomoxis annularis & White Crappie & $\mathrm{N}$ & $0,1+$ & $1+$ & $1+$ & $\mathrm{Ph}$ & $\mathrm{R}$ & & 48 \\
\hline Perca flavescens & Yellow Perch & $\mathrm{N}$ & $0,1+$ & 0 & & $\mathrm{Pl}$ & $\mathrm{R}$ & & 11 \\
\hline Etheostoma nigrum & Johnny Darter & $\mathrm{N}$ & $0,1+$ & 0 & $0,1+$ & $\mathrm{Sp}$ & $\mathrm{C}$ & 1.1 & 5 \\
\hline Percina caprodes & Logperch & $\mathrm{N}$ & $0,1+$ & $0,1+$ & & Ps & $\mathrm{R}$ & & 14 \\
\hline Percina maculata & Blackside Darter & $\mathrm{N}$ & $1+$ & & $1+$ & $\mathrm{Li}$ & $\mathrm{R}$ & & \\
\hline Labidesthes sicculus & Brook Silverside & $\mathrm{N}$ & $0,1+$ & 0 & & $\mathrm{Pl}$ & $\mathrm{C}$ & 6.3 & 5 \\
\hline Aplodinotus grunniens & Freshwater Drum & $\mathrm{N}$ & $0,1+$ & & & $\mathrm{Pe}$ & $\mathrm{R}$ & & 3 \\
\hline Neogobius melanostomus & Round Goby & $\mathrm{I}$ & $1+$ & & & $\mathrm{Sp}$ & $\mathrm{R}$ & & \\
\hline
\end{tabular}

tributed just 3\% to the overall catch. Conversely, Logperch, Smallmouth Bass, and Longnose Gar, found in 1995 were not caught in 1994; these fishes were caught in small number, usually on a single date. Gizzard Shad (25.4\%), Brook Silverside (21.9\%), Orangespotted Sunfish (19.2\%), and Bluegill (13.9\% of total catch) were most abundant of 36 species and 3055 specimens collected in 1994. Frequency of occurrence of these four taxa was also highest $\left(\mathrm{f}_{\mathrm{o}}=0.8-1.0\right)$ throughout the sampling period. Orangespotted Sunfish and Spottail Shiners were the only adult fishes collected consistently. 
TABle 3. Percent contribution, seasonality, and frequency of occurrence (parentheses) of dominant fishes at three collection sites in River Canard, 1994-1995.

\begin{tabular}{|c|c|c|c|c|c|c|c|c|}
\hline \multirow{2}{*}{$\begin{array}{l}\text { Species } \\
\text { Gizzard Shad }\end{array}$} & \multicolumn{2}{|c|}{$\begin{array}{c}\text { Lower } \\
1994\end{array}$} & \multicolumn{2}{|l|}{1995} & \multicolumn{2}{|l|}{ Mid } & \multicolumn{2}{|c|}{ Upper } \\
\hline & 25.4 & $\begin{array}{l}\text { June-November } \\
\text { (1.0) }\end{array}$ & 80.7 & $\begin{array}{l}\text { June-October } \\
(0.9)\end{array}$ & 78.5 & $\begin{array}{l}\text { June-September } \\
(0.9)\end{array}$ & 6.2 & $\begin{array}{l}\text { June, August, } \\
\text { September (0.3) }\end{array}$ \\
\hline Emerald Shiner & 5.4 & $\begin{array}{l}\text { June-November } \\
(0.2)\end{array}$ & 0.6 & June-August (0.4) & 8.1 & June-July (0.3) & & \\
\hline Common Carp & 0.2 & June-July (0.3) & 0.5 & June-August (0.4) & 2.9 & June-July (0.4) & & June (0.2) \\
\hline Bluntnose Minnow & 1.2 & $\begin{array}{l}\text { June-August } \\
(0.6)\end{array}$ & 0.2 & $\begin{array}{l}\text { June-July, } \\
\text { September }(0.5)\end{array}$ & & June $(0.1)$ & 13.7 & $\begin{array}{l}\text { June-August } \\
(0.6)\end{array}$ \\
\hline Orangespotted Sunfish & 19.2 & $\begin{array}{l}\text { June-November } \\
(0.9)\end{array}$ & 0.6 & $\begin{array}{l}\text { June-August } \\
(0.6)\end{array}$ & 1.2 & $\begin{array}{l}\text { June-July } \\
(0.2)\end{array}$ & 68.3 & $\begin{array}{l}\text { June-September } \\
(0.6)\end{array}$ \\
\hline Bluegill & 13.9 & $\begin{array}{l}\text { June-November } \\
(0.8)\end{array}$ & 3.1 & $\begin{array}{l}\text { June-September } \\
(0.8)\end{array}$ & 2.9 & $\begin{array}{l}\text { June-July } \\
(0.4)\end{array}$ & 1.8 & $\begin{array}{l}\text { June, September } \\
(0.2)\end{array}$ \\
\hline Brook Silverside & 21.9 & $\begin{array}{l}\text { June-November } \\
(0.9)\end{array}$ & 10.1 & $\begin{array}{l}\text { June-September } \\
(0.6)\end{array}$ & & July (0.1) & & \\
\hline Total catch & 2671 & & 3452 & & 1089 & & 1532 & \\
\hline
\end{tabular}

TABLE 4. Comparitive growth in length of eight species of age-0 fishes in the Great Lakes basin.

\begin{tabular}{|c|c|c|c|c|c|}
\hline \multirow[t]{2}{*}{ Species } & \multirow[b]{2}{*}{ System } & \multirow[b]{2}{*}{ Year } & \multicolumn{2}{|c|}{ Mid-month TL (mm) } & \multirow[b]{2}{*}{ Reference } \\
\hline & & & August & September & \\
\hline \multirow[t]{6}{*}{ Gizzard Shad } & Lotic & 1994 & $62.2(42)$ & $80.2(66)$ & Present study \\
\hline & & 1995 & $69.7(26)$ & 77.8 (19) & \\
\hline & Lotic & 1994 & $48.5(23)$ & $72.3(27)$ & Leslie et al. 1999 \\
\hline & Lentic & 1994 & $45.7(15)$ & $58.2(26)$ & Leslie and Timmins $1998 \mathrm{~b}$ \\
\hline & Lentic & 1990 & $51.9(20)$ & $74.0(18)$ & Leslie and Timmins 1998b \\
\hline & Lacustrine & 1983 & $32.5(82)$ & & Leslie and Timmins 1993 \\
\hline \multirow[t]{4}{*}{ Brook Silverside } & Lotic & 1994 & $42.6(25)$ & & Present study \\
\hline & Lotic & 1994 & $44.6(24)$ & & Leslie et al. 1999 \\
\hline & Lentic & 1994 & $46.0(23)$ & & Leslie and Timmins 1998b \\
\hline & Lacustrine & 1983 & $43.0(59)$ & & Leslie and Timmins 1993 \\
\hline \multirow[t]{4}{*}{ Emerald Shiner } & Lotic & 1994 & $33.9(35)$ & $43.5(14)$ & Present study \\
\hline & Lotic & 1994 & $34.7(43)$ & $41.5(26)$ & Leslie et al. 1999 \\
\hline & Lotic & 1994 & 44.9 (39) & $48.2(32)$ & Leslie et al. 1999 \\
\hline & Lacustrine & 1994 & & $42.4(21)$ & Leslie and Timmins 1998c \\
\hline \multirow[t]{5}{*}{ Bluntnose Minnow } & Lotic & 1994 & $26.0(15)$ & & Present study \\
\hline & & 1995 & $29.5(24)$ & & \\
\hline & Lotic & 1994 & $45.3(8)$ & $50.2(20)$ & Leslie et al. 1999 \\
\hline & Lacustrine & 1977 & $36.4(72)$ & $51.3(79)$ & Keast and Eadie 1984 \\
\hline & Lacustrine & 1998 & & $29.7(55)$ & Leslie and Timmins 2002 \\
\hline \multirow[t]{3}{*}{ Orangespotted Sunfish } & Lotic & 1994 & & $38.1(23)$ & Present study \\
\hline & & 1995 & & $34.6(15)$ & \\
\hline & Lentic & 1990 & & $36.5(22)$ & Leslie and Timmins 1998b \\
\hline \multirow[t]{5}{*}{ Bluegill } & Lotic & 1994 & $30.6(37)$ & $36.9(26)$ & Present study \\
\hline & & 1995 & $31.0(15)$ & $34.4(48)$ & \\
\hline & Lotic & 1994 & & $33.3(67)$ & Leslie et al. 1999 \\
\hline & Lacustrine & 1977 & $16.4(56)$ & $26.7(35)$ & Keast and Eadie 1984 \\
\hline & Lentic & 1990 & & 28.7 (19) & Leslie and Timmins 1998b \\
\hline \multirow[t]{3}{*}{ Black Crappie } & Lotic & 1994 & $56.7(26)$ & $59.9(15)$ & Present study \\
\hline & & 1995 & & $62.2(29)$ & \\
\hline & Lotic & 1994 & $55.5(12)$ & $61.2(17)$ & Leslie et al. 1999 \\
\hline \multirow[t]{3}{*}{ Largemouth Bass } & Lotic & 1994 & & $91.9(10)$ & Present study \\
\hline & & 1995 & & $72.6(10)$ & \\
\hline & Lacustrine & 1977 & & $48.6(89)$ & Keast and Eadie 1984 \\
\hline
\end{tabular}




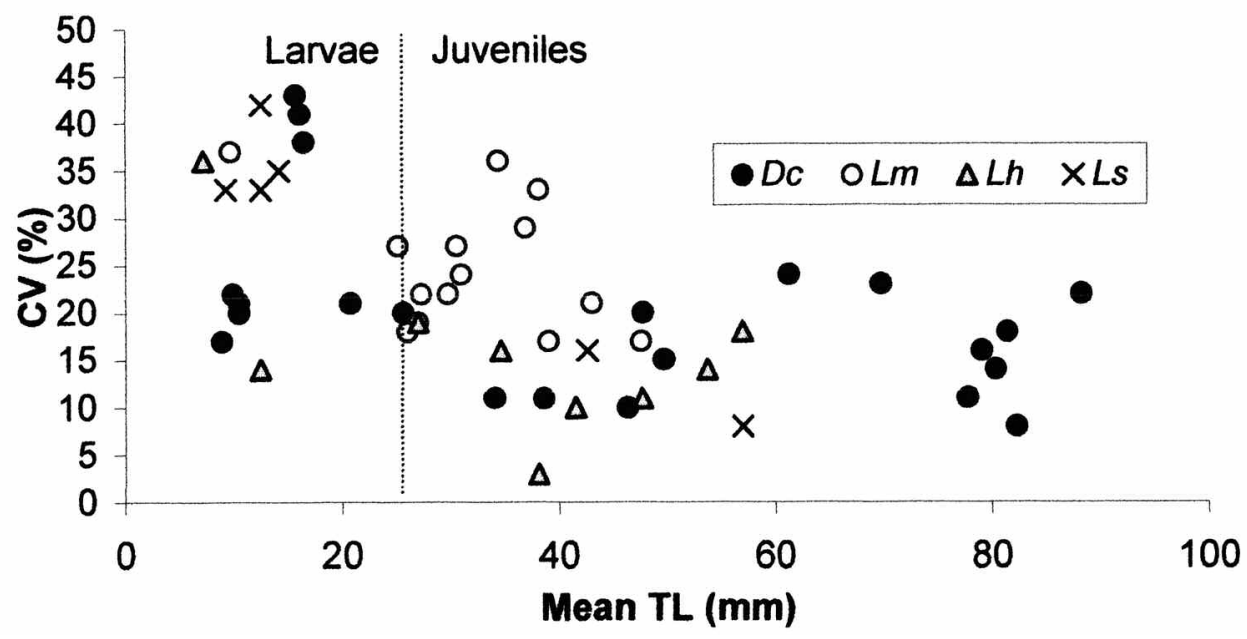

FIgURE 4. Coefficient of variation in mean total lengths of Gizzard Shad (Dc), Bluegill $(\mathrm{Lm})$, Orangespotted Sunfish ( $L h)$, and Brook Silverside $(L s)$ in River Canard, Ontario. Vertical line delineates larvae $(<25 \mathrm{~mm})$ and age 0 juveniles.

Total catch in 1995 (8771 fishes, 26 species) was dominated by Gizzard Shad $(80.7 \%)$, Brook Silverside $(10.1 \%)$ and Bluegill (3.1\%). No fish larvae were found in April or May. Largest catches of Gizzard Shad (4$5 \mathrm{~mm}$ ), Orangespotted Sunfish and Bluegill were made in late June. Spottail Shiner, Bluegill, Black Crappie, Brook Silverside, Bluntnose Minnow, and Largemouth Bass were considered "residents" based on high persistence. As in 1994, adult Orangespotted Sunfish and Spottail Shiner were collected on most dates.

Plankton net hauls in open water collected 5280 age 0 fishes (10 species) in mid-June, 1995. None of these species was unique to the assemblage collected with beach seines. However, because Freshwater Drum spawn in open water where semi-buoyant eggs drift with currents, the plankton net collected more eggs and free embryos $(3.7 \pm 0.4 \mathrm{~mm} ; \mathrm{N}=13)$ than did the beach seine. Total catch was dominated by Gizzard Shad (97.3\%), Common Carp (1.0\%) and Freshwater Drum $(0.6 \%)$. Mean fish density varied widely among hauls and dates. For example, mean densities ranged from 4201 to $6390 / 100 \mathrm{~m}^{3}$ and 1199 to $2419 / 100 \mathrm{~m}^{3}$ on 11 June and 12 June, respectively.

\section{Growth of most-abundant fishes}

Lengths near the end of first year growth differed according to species. Bluntnose Minnow and Brook Silverside were the only fishes that nearly attained adult length in late summer. Gizzard Shad, Largemouth Bass, and Black Crappie are among the largest fishes in the assemblage (Table 4). These species achieved 12 to 25 times their length at hatch, whereas small-bodied fishes (e.g., cyprinids, Brook Silverside) increased 6 to 8-fold hatch size. Coefficient of variation (CV) in mean total length of four most-abundant species was highest in larvae $(<25 \mathrm{~mm})$ and tended to decrease with size (Figure 4). In general, highest variability in species' lengths coincided with overall peak abundance in midJune to mid-July.

\section{Discussion}

The assemblage of age 0 fishes (42 taxa) may be considered a cyprinid-centrarchid-clupeid-atherinid complex numerically representing $91 \%$ of the catch (Table 2) and $63 \%$ of a taxocene strongly dominated by a planktivore, Gizzard Shad. Lithophils (non-guarding and guarding) and phytophils otherwise predominate in constant turbid water and shoreline with limited diversification. Larvae of these guilds are initially photophobic (Mann 1996) and in this respect, perhaps tolerant of turbid conditions. However, the presence of most species in River Canard belies spawning requirements for gravel, macrophytes, and clear water. Orangespotted Sunfish selects shallow, silt-laden water (Noltie and Beletz 1984) and is an appropriate indicator species, contrary to Bluegill, which usually chooses vegetated shallows in clear water (Trautman 1981).

Similarly, Emerald Shiner, a pelagophil, was common and abundant at the middle site and in the lower reach. It was also found, albeit in low numbers, in adjacent watersheds, shore areas, and streams in south-east Lake St. Clair and western Lake Erie (Noltie and Beletz 1984; Leslie and Timmins 1998a,b,c; Leslie et al. 1999). Undoubtedly, this is a plastic species with considerable ecological amplitude, as is the Bluntnose Minnow. Brook Silverside, another common taxon, usually spawns on emergent or floating leaf plants, hence its absence in the upper reach and presence in the lower reach. The assemblage in the lower reach represented many ecological guilds dominated by persistent fishes, such as Gizzard Shad, Brook Silverside, Emerald Shin- 
er, Orangespotted Sunfish, and Bluegill. Gizzard Shad spawned in the upper reach and undoubtedly further upstream in connecting minor drainage systems, where larvae are found in vast number (Leslie and Timmins 1990).

Most fishes expanded their ecological limits with growth and development. Species turnover was typically high and relatively few taxa occurred simultaneously at the three sites. These findings concur with those in similar studies in adjacent watersheds of the St. Clair and western Lake Erie flatlands (Leslie and Timmins 1990, 1998a; Leslie et al. 1999). Commonly, species richness and abundance are highly correlated with macrophyte diversity and shallow marginal habitat that provides refuge against predators (Eadie and Keast 1984; Petering and Johnson 1991; Copp and Mann 1993; Mann 1996; Duncan et al. 2001). Sinuous and sluggish, River Canard is devoid of vegetated backwaters that enhance diversity, spawning and nursery habitats, and food availability (Oberdorff et al. 1993). Nevertheless, Pugnose Minnow, Pugnose Shiner, and Central Stoneroller, sensitive to turbid waters, tolerated adverse conditions, at least temporarily.

Environmental variables and species numbers were not related with seasonal abundance (Table 1; Figure 3 ). Number and abundance of age 0 fishes in the lower reach were likely augmented by continuous larval drift, individuals purged from upstream during spates, and migrants from connecting drainage ditches and the Detroit River. Although River Canard appears to be a harsh environment for fishes during early ontogeny, large numbers of taxa have adapted to, if not thrived, in this highly perturbed system. Abiotic and biotic factors influence rate of growth during early development, and hence, recruitment. Total length attained by recruits near end of summer may well reflect these factors, providing sampling does not seriously bias size distributions of any given species (Bayley and Dowling 1993; Leslie and Timmins 1994).

With few exceptions, dominants were much the same length as those recorded elsewhere in the ecoregion (Table 4). For example, Gizzard Shad and Emerald Shiner reached autumnal lengths comparable to those in turbid streams and bays but less than in waters of higher quality, transparency and productivity, such as the Detroit River (Leslie et al. 1999). Orangespotted Sunfish were slightly longer in River Canard than reported for specimens in lowland Ohio streams (Trautman 1981), whereas Bluntnose Minnows were smaller than counterparts in the St. Clair region. Similarly, Bluegill and Brook Silverside attained lengths similar to those of species elsewhere in the lower Great Lakes basin (Trautman 1981; Leslie and Timmins 1990; 1998a,b; Leslie et al. 1999). Size range relative to mean total length of cyprinids and other small species was usually much lower for age 0 juveniles than for larvae, as was overall coefficient of variation in length. Sampling bias, predation, and natural mortality of small- est fish may effect a reduction in variability in length as growth reaches a plateau.

The diverse age 0 fish taxocene in River Canard is represented by all reproductive guilds in Canada. Yet, according to descriptions in Scott and Crossman (1973) and Trautman (1981), fewer than 25\% of species listed in Table 2 normally utilise environmental conditions that prevail in the river. Apparently, assignment of certain species to a specific reproductive or ecological guild requires revision for Great Lakes as well as European freshwater fishes (Mann 1996). Perhaps these realities reflect our limited knowledge of fish ecology in lowland streams.

\section{Acknowledgments}

We thank Susan Saunders of Water Surveys of Canada, Burlington, for flow data for River Canard, R.E. Dermott for assistance in the field, and several anonymous reviewers.

\section{Literature Cited}

Balon, E. K. 1975. Reproductive guilds of fishes: a proposal and definition. Journal of Fisheries Research Board Canada 32: 821-864.

Bayley, P. B., and D. C. Dowling. 1993. The effect of habitat in biasing fish abundance and species richness estimates when using various sampling methods in streams. Polskie Archiwum Hydrobiologii 40: 5-14.

Campbell, R. R. 2001. Rare and endangered fishes and marine mammals of Canada; COSEWIC Fish and Marine Mammal Subcommittee Status Reports XIV. Canadian Field-Naturalist 115: 564-572.

Canada Department of Fisheries and Oceans (DFO). 1984. Canada fish habitat law. Catalogue FS 23-40/1983E. Ottawa.

Chapman, L. J., and D. F. Putnam. 1984. The physiography of southern Ontario. Ontario Geological Survey Special Volume 2. Toronto.

Copp, G. H., and R. H. K. Mann. 1993. Comparative growth and diet of tench Tinca tinca (L.) larvae and juveniles from river floodplain biotopes in France and England. Ecology Freshwater Fishes 2: 58-66.

Duncan, A., J. Kubecka, S. Kett, N. Hanna, and J. Skeldon. 2001. Habitats of 0+ fry in an English lowland river. Archivum für Hydrobiologie 12 Supplement 135/2-4: 153-171.

Eadie, J., and A. Keast. 1984. Resource heterogeneity and fish species diversity in lakes. Canadian Journal of Zoology 62: 1689-1695.

Haffner, G. D. 1992. Editorial: Research and environmental policy. Journal of Great Lakes Research 18: 521.

Herdendorf, C. E. 1987. The ecology of the coastal marshes of western Lake Erie: a community profile. U.S. Fish and Wildlife Service Biological Report 85(7.9).

Keast, A., and J. Eadie. 1984. Growth in the first summer of life: a comparison of nine co-occuring fish species. Canadian Journal of Zoology 62: 1242-1250.

Leslie, J. K., J. E. Moore, W. H. Hyatt, and C. A. Timmins. 1983. Seine for sampling larval fish in shallow water. Progressive Fish-Culturist 45: 130-131.

Leslie, J. K., and C. A. Timmins. 1990. The community of young fish in drainage ditches in southwestern Ontario. Archivum für Hydrobiologie 118: 227-240. 
Leslie, J. K., and C. A.Timmins. 1993. Distribution, density, and growth of young-of-the-year fishes in Mitchell Bay, Lake St. Clair. Canadian Journal of Zoology 71: 1153-1160.

Leslie, J. K., and C. A. Timmins. 1994. Habitat distribution in relation to sample collections of young of year fishes. Polskie Archiwum Hydrobiologii 41: 301-310.

Leslie, J. K., and C. A. Timmins. 1998a. Fish reproduction and distribution in a small tributary of Lake St. Clair. Canadian Technical Report of Fisheries and Aquatic Sciences 2253.

Leslie, J. K., and C. A. Timmins. 1998b. Age 0+ fish occurrence in modified habitat in south-western Ontario. Canadian Technical Report of Fisheries and Aquatic Sciences 2219.

Leslie, J. K., and C. A. Timmins. 1998c. Seasonality of fish larvae in surf zone and tributary of Lake Erie: a comparison. Canadian Technical Report of Fisheries and Aquatic Sciences 2197.

Leslie J. K., and C. A. Timmins. 2002. Fishes of the shallow littoral zone of Fathom Five National Marine Park. Pages 167-178 in Ecology, culture and conservation of a protected area: Fathom Five National Marine Park, Canada. Edited by S. Parker and M. Munawar. Backhuys Publishing, Leiden, The Netherlands.

Leslie, J. K., C. A. Timmins, and A. Pachkevitch. 1999. Age 0 fish assemblages in proximate systems: the Detroit River and Cedar Creek. Canadian Technical Report of Fisheries and Aquatic Sciences 2279.

Mandrak, N. E., and E. J. Crossman. 1992. A checklist of Ontario freshwater fishes. Royal Ontario Museum. Toronto, Ontario.

Mann, R. K. H. 1996. Environmental requirements of European non-salmonid fish in rivers. Hydrobiologia 323: 223235.
Noltie, D. B., and F. Beletz. 1984. Range extension of the Orangespotted Sunfish, Lepomis humilis, to the Canard River, Essex County, Ontario. Canadian Field-Naturalist. 98: 494-496.

Oberdorff, T., E. Guibert, and J-C Lucchetta. 1993. Patterns of fish species richness in the Seine River basin, France. Hydrobiologia 259: 157-167.

Ontario Ministry of Environment and Energy. (MOEE). 1994. Water quality data Ontario lakes and streams 1990. Volume XXXVI: Southwestern region.

Oosting, H. J. 1956. The study of plant communities. An introduction to plant ecology. W. H. Freeman Co. San Francisco, California.

Petering, R. W., and D. L. Johnson. 1991. Distribution of fish larvae among artificial vegetation in a diked Lake Erie wetland. Wetlands 11: 123-138.

Przybylski, M. 1994. Are the fish communities persistent and stable in European rivers? Polskie Archiwum Hydrobiologii 41: 365-375.

Scott, W. B., and E. J. Crossman. 1973. Freshwater fishes of Canada. Bulletin of Fisheries Research Board of Canada. (184).

Trautman, M. B. 1981. The fishes of Ohio. Ohio State University Press, Columbus, Ohio.

Winner, J. M., and J. P. Hartt. 1969. A limnological study of River Canard, Essex County, Ontario. Pages 103-116 in Proceedings $12^{\text {th }}$ Conference of Great Lakes Research. International Association of Great Lakes Research.

Received 20 May 2003

Accepted 22 November 2004 